\title{
AFFORDABLE ANNOTATION OF THE MOBILE APP REVIEWS
}

\author{
MAREK GRÁC - MARKÉTA MASOPUSTOVÁ - MARIE VALÍČKOVÁ \\ Department of Czech Language, Faculty of Arts, Masaryk University, Brno, \\ Czech Republic
}

GRÁC, Marek - MASOPUSTOVÁ, Markéta - VALÍČKOVÁ, Marie: Affordable annotation of the mobile app reviews. Journal of Linguistics, 2019, Vol. 70, No 2, pp. 491 $-497$.

\begin{abstract}
This paper focuses on the use-case study of the annotation of the mobile app reviews from Google Play and Apple Store. These annotations of sentiment polarity were created for later use in the automatic processing based on machine learning. This should solve some of the problems encountered in the previous analyses of the Czech language where data assumptions play a greater role than annotation itself (due to the financial constraints). Our proposal shows that some of the assumptions used for English do not apply to Czech and that it is possible to annotate such data without extensive financing.
\end{abstract}

Keywords: sentiment polarity, topics analysis, annotation

\section{INTRODUCTION}

The Internet expansion was followed by various business models, including online stores, applications and other online services. The impact of the user feedback (and virality) might be so significant that it might make the difference between success and failure. In order to process such feedback correctly, it is necessary to monitor discussions and reply to users. In case of a very small user base, it is possible to read every single comment by an expert, but such approach is too naïve for applications with a bigger audience. In such case, it is necessary to do some form of data aggregation which is later processed by an expert. This aggregation might be done manually or automatically. However, automatic supervised methods usually require a non-trivial amount of high-quality annotated data, so manual annotation seems more reasonable as a first step in the majority of the projects.

The issue of niche languages like Czech is that manually annotated data are usually not available and therefore we need to train our model on the similar (available) data. In the previous projects focusing on the Czech language, the models were trained on the data that allow their automatic classification ([1], [2]), e.g. price comparison website Heureka containing reviews of thousands of different products from users who assess positive and negative sides of the product. Such "pre-annotated" data limits the 
scope of our research because we have to rely on the existing data or data derived from it. In chapter 4, we will demonstrate that some of these assumptions might not be precise enough in comparison with the annotated data.

In our project, we chose to manually annotate the data by experts and not to rely on the publicly available data. Our work focuses on sentiment polarity (and topics analysis $\left.{ }^{1}\right)$. Because these two areas can help businesses understand the needs of customers as much as possible, it is expectable they will prefer an automatic (and also cheaper) solution in the future, but even partial results can be used during the annotating phase. The aim of this article is to provide insights into the annotation and compare our results with previously widely accepted assumptions.

\section{STATE OF THE ART}

Analysis of the sentiment and particularly the sentiment polarity is a heavily investigated area. The best approaches usually compete in SemEval challenges [3], where the sentiment analysis on Twitter is one of the challenges. The situation of the Czech language is very close to other Slavic languages. There are several proprietary technologies from global and local companies that have never been properly benchmarked and several smaller published projects.

The most notable projects are [2] where the corpus of 10,000 Facebook posts was annotated by two annotators, Cohen's $\kappa 0.66$ on the document-level annotation. Most of these conflicts were cases of disagreement between neutral and bipolar. Preannotated data where sentiment polarity is not explicit are in the order of magnitude larger, but their quality is questionable.

The other important source for the sentiment analysis in Czech is [1], where the polarity sentiment is split into five categories: negative, non-negative, neutral, nonpositive and positive. Even though sentiment analysis can be done on various levels, it was shown that document level text analysis relies heavily on the redundancy and various hints, which is very difficult for automatic analysis. On the sentence-level, the situation is simpler but we need to derive the overall polarity based on the polarity of particular sentences. This derivation, in general, is a complex problem as one single negative sentence can override several of the positive ones. The longer the document, the harder the problem.

In their project, they used three different datasets. The first dataset was compiled from 12 randomly chosen opinion articles from a news server aktualne.cz. Two annotators annotated 410 segments of texts (6.868 words and 1.935 unique lemmas) with the result of Cohen's $\kappa 0.63$. The second dataset was compiled from the reviews of movies on csfd.cz. It was created to compare the outcome with the previous one. In total, there were 405 segments with the result of Cohen's $\kappa 0.66$. The last dataset

\footnotetext{
${ }^{1}$ However, the topics analysis is not the subject of this article.
} 
was not annotated by the annotators. It was taken from a retail server Mall.cz which also includes user product reviews.

\section{DATA AND ANNOTATION PROCESS}

In our project, we were working with the reviews of the $\mathrm{B} 2 \mathrm{C}$ (business to customers) mobile applications of Czech companies. Data were obtained from public sources from both major platforms: Google Play and Apple Store. Only reviews written in Czech were annotated and they will be used as training data. All reviews were also anonymized as identification of the author is not important for our project. An average review consists of a few sentences only, so texts are relatively short (Figure 1) and the majority focuses on the application itself. It makes our situation quite similar to the analysis of tweets that are heavily investigated by ([4], [5]).

Each review was annotated for sentiment analysis on the sentence-level. Our first attempt was to annotate on the segment-level but we found out that even if we can obtain acceptable inter-annotator agreement (IAA), the process is too expensive for the annotation. Our annotators were able to annotate 250 reviews on the segmentlevel in 10 hours, while the annotation of the same reviews on the sentence-level was finished in 4 hours. Annotation on the document-level was rejected directly based on the results of [6].

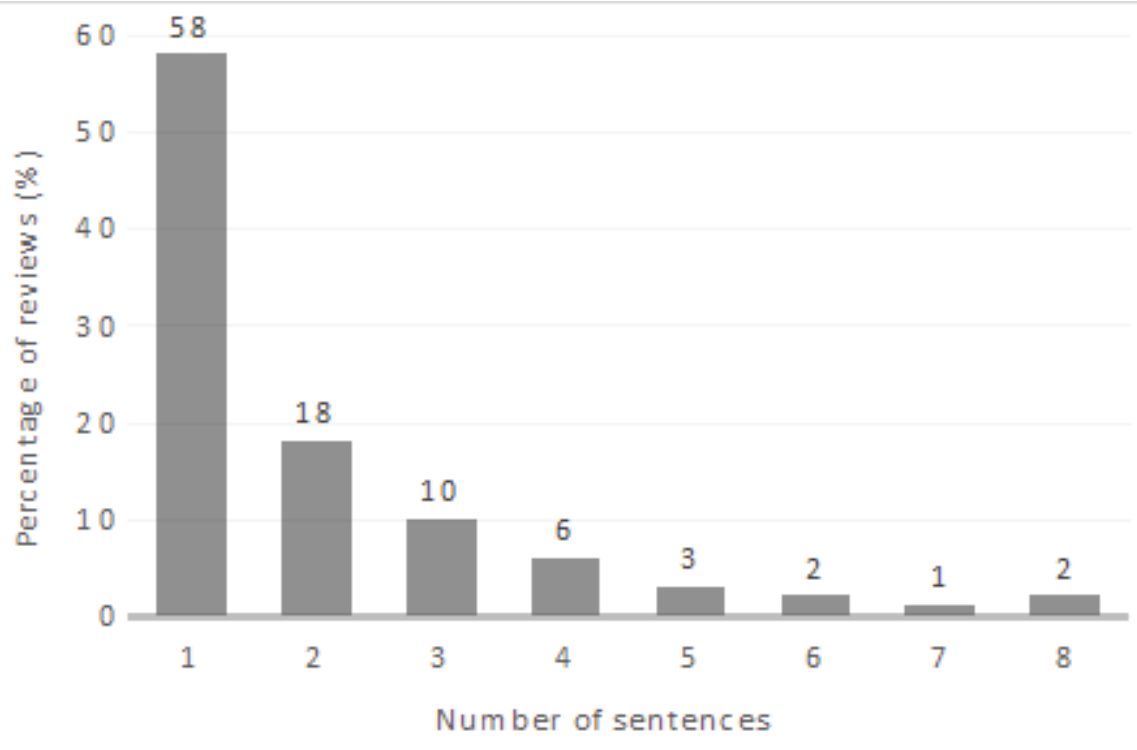

Fig. 1. A number of sentences in reviews 
Every review is automatically split into sentences by using the Czech module of Punkt system [7] that can be edited by annotators to fulfil the annotation guidelines. Punkt language module has built-in language model which was used without modifications. Our original assumption was that it will be necessary to retrain the model to suit our domain better. We found out that the majority of the errors end in a situation where sentences are not split correctly. Those situations usually contain symbol ... where it is not clear where sentence boundaries are. Annotators work with each sentence separately, therefore, there is a chance of missing some inter-sentence information when a review is split into too many parts.

Such annotation of the training data is directly usable for business owners even when only part of the data are annotated. Customers can use it almost immediately and it makes the industry more open to funding such research projects.

4 Annotation of the Sentiment Polarity

The reviews are rather short; the majority of them contains only up to three sentences. Annotators annotate each of them with a preferred sentiment. After discussion, we have selected the simplest solution even though we see the benefits of using non-positive or weakly positive labels. Our primary interest was high consistency (IAA) and annotation cost. These labels were selected: positive, neutral, negative and mixed $^{2}$. At the first iteration, we were able to reach Cohen's $\kappa 0.58$ (on sentence-level), which is close to the published numbers in ([1], [2]). In the confusion matrix in Table 1, the differences between annotators are observable.

\begin{tabular}{|c|c|c|c|c|}
\hline & + & \pm & - & 0 \\
\hline+ & $\mathbf{1 8}$ & 0 & 0 & 0 \\
\hline \pm & 0 & $\mathbf{3 0}$ & 7 & 1 \\
\hline- & 0 & 4 & $\mathbf{1 2 5}$ & 2 \\
\hline 0 & 1 & 2 & 35 & $\mathbf{5}$ \\
\hline
\end{tabular}

Tab. 1. Inter-annotator agreement

As sentiment analysis is subjective even when annotation guideline is used, the small differences are acceptable. The only relevant issues are 35 (out of 250) differences where the first annotator chose neutral label but the other one preferred negative one. After investigating these cases, we found out that the majority of the problems was caused by very short reviews that contained neutral words only. One of the annotators was annotating what was written, but the other one was annotating what the user meant, e. g. Reklama 'Advertisement' means that there are too many of the ads in the application. We shifted the annotation to annotate what the user meant even if it is likely to decrease the results of our project. This is a different kind of a problem than reported in the previous papers where discrepancies occur mainly between neutral and mixed polarity.

${ }^{2}$ Mixed polarity is used when part of the sentence is positive and the other part is negative. 
After we have annotated sentences, it is possible to derive sentiment of the whole document/review. It is rather difficult to differentiate the sentiment polarity on a whole document (e.g. on a news article). Thanks to our short reviews, we can select the resulting polarity based on the existence of at least one positive/negative sentences (where mixed sentiment is count as both positive and negative polarity, and neutral sentences are ignored). This approach is not reasonable in longer documents because mixed polarity will prevail as documents usually contain at least one positive and negative sentence. In our case, this does not occur. The number of documents with mixed polarity ranges between 15 and $26 \%$ in each set of annotated data.

In order to manage the labels better, we added one more label: irony, for figurative meanings, irony and sarcasm for the Czech user is rather ironic and sarcastic. Usually, it is not noticeable from just one sentence (e.g. Je to přesně tak, jakmá být. K nejhorší bances nejhoršími a nejdražšimi službami patříneodmyslitelně i bezkonkurenčně nejhorši aplikace. 'It's exactly as it should be. One of the worst banks with the worst and most expensive services has inherently the worst application.'). We annotate these reviews but they are not used for training/testing.

In other projects [1], the author took a huge amount of data from the evaluation sites or online stores. They rely on the quantity of data annotated by random users and take the text written in "plus" section as positive and the text written in "minus" section as negative. However, the reviews often contain irrelevant information, at least in the case of the Czech reviews (e.g. under "minus" section is frequently used word nic 'nothing' Nevím. 'I don't know.' or Nic mě nenapadá. 'Nothing comes on my mind'). It is important to take into consideration that some features can be positive for one product, but negative for the other (e.g. loudness could be beneficial in the case of a mobile phone but unpleasant for a hover). Thus, this is not the best approach.

Another issue of this data is that we rely on the assumption that reviews with a high rating are positive reviews and with a low rating are negative. We found out (Figure 2) that this assumption is valid in the case of negative ratings, but even reviews with a perfect rating (5 stars out of 5) are positive only in approx. $60 \%$ of cases. The rest of the reviews is evenly split between negative, mixed and neutral reviews. It is possible that a similar pattern can be seen also on different data. It could explain a gap between the results of the Czech and English language.

\section{CONCLUSION}

This article presented the annotation of the sentiment polarity on the reviews of the mobile applications of the given Czech B2C applications. We are annotating more than thousand reviews on a monthly basis and we are happy that we can offer part of them under CC-BY-NC license for other researchers. Those reviews are annotated on a sentence-level for the sentiment polarity and are available on https://github.com/bedeep/mobile-app-sentiment-data. 
In our future work, we will focus on automatic detection of the sentiment polarity of a text. We plan to re-test existing approaches for the Czech language and re-implement state of the art techniques in the near future. If any of these approaches succeed, the annotators will switch from adding information to the sentences to the validation of proposed information. This should help us obtain more data even cheaper.

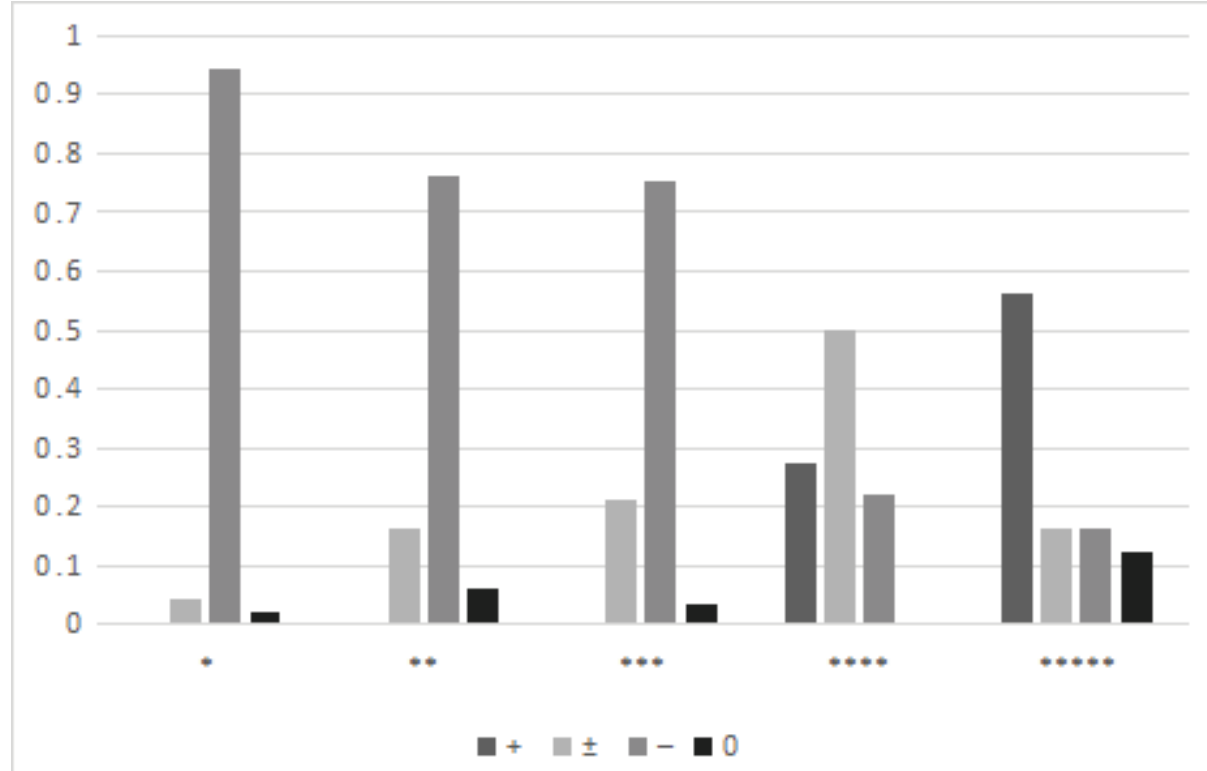

Fig. 2. The relation between rating and sentiment.

References

[1] Veselovská, K. (2017). Sentiment analysis on Czech. Praha, Karolinum.

[2] Habernal, I., Ptáček, T., and Steinberger, J. (2013). Sentiment Analysis in Czech Social Media Using Supervised Machine Learning. In Proceedings of the $4^{\text {th }}$ Workshop on Computational Approaches to Subjectivity, Sentiment and Social Media Analysis, pages 6574, Atlanta, Georgia: Association for Computational Linguistics.

[3] Rosenthal, S., Farra, N., and Nakov, P. (2017). SemEval-2017 Task 4: Sentiment Analysis in Twitter. In Proceedings of the $11^{\text {th }}$ International Workshop on Semantic Evaluation (SemEval-2017), pages 502-518, Vancouver, Canada: Association for Computational Linguistics.

[4] Anta, A. F., Chiroque, L. N., Morere, P., and Santos, A. (2013). Sentiment Analysis and Topic Detection of Spanish Tweets: A Comparative Study of NLP Techniques. In Procesamiento del lenguaje natural, pages 45-52. 
[5] Agarwal, A., Xie, B., Vovsha, I., Rambow, O., and Passonneau, R. (2011). Sentiment Analysis of Twitter Data. In Proceedings of the Workshop on Language in Social Media (LSM 2011), pages 30-38, Portland, Oregon: Association for Computational Linguistics.

[6] Wiegand, M., and Dietrich, K. (2009). The role of knowledge-based features in polarity classification at sentence level. In Proceedings of the $22^{\text {nd }}$ International FLAIRS Conference.

[7] Kiss, T., and Strunk, J. (2006). Unsupervised Multilingual Sentence Boundary Detection. In Computational Linguistics, pages 485-525. 